\title{
Mechanistic Basis for the Failure of Cone Transducin to Translocate: Why Cones Are Never Blinded by Light
}

\author{
Ekaterina S. Lobanova, ${ }^{1}$ Rolf Herrmann, ${ }^{1}$ Stella Finkelstein, ${ }^{1}$ Boris Reidel, ${ }^{1}$ Nikolai P. Skiba, ${ }^{1}$ Wen-Tao Deng, ${ }^{2}$ \\ Rebecca Jo, ${ }^{3}$ Ellen R. Weiss, ${ }^{3}$ William W. Hauswirth,${ }^{2}$ and Vadim Y. Arshavsky ${ }^{1}$ \\ ${ }^{1}$ Albert Eye Research Institute, Duke University, Durham, North Carolina 27710, ${ }^{2}$ Department of Ophthalmology, University of Florida, Gainesville, Florida \\ 32610, and ${ }^{3}$ Department of Cell and Developmental Biology, The University of North Carolina at Chapel Hill, Chapel Hill, North Carolina 27599
}

The remarkable ability of our vision to function under ever-changing conditions of ambient illumination is mediated by multiple molecular mechanisms regulating the light sensitivity of rods and cones. One such mechanism involves massive translocation of signaling proteins, including the G-protein transducin, into and out of the light-sensitive photoreceptor outer segment compartment. Transducin translocation extends the operating range of rods, but in cones transducin never translocates, which is puzzling because cones typically function in much brighter light than rods. Using genetically manipulated mice in which the rates of transducin activation and inactivation were altered, we demonstrate that, like in rods, transducin translocation in cones can be triggered when transducin activation exceeds a critical level, essentially saturating the photoresponse. However, this level is never achieved in wild-type cones: their superior ability to tightly control the rates of transducin activation and inactivation, responsible for avoiding saturation by light, also accounts for the prevention of transducin translocation at any light intensity.

\section{Introduction}

Vertebrate vision has a remarkable ability to adapt its speed and sensitivity to the dramatic changes in ambient light intensity that take place during a normal diurnal cycle (Rodieck, 1998). This is achieved in part by the two types of photoreceptors, rods and cones, optimized to operate under different illumination conditions. Rods are extremely sensitive to light and function as ultimate singlephoton detectors. However, their overall contribution to visual experience is markedly diminished at the light intensities sufficient for color perception and their photoresponses could be completely saturated in bright daylight. Cones have much lower light sensitivity than rods, but they produce faster responses and adapt to light more efficiently. These properties allow cones to avoid response saturation under any natural conditions of illumination and to provide higher temporal resolution of vision than rods.

Persistent light exposure induces adaptive changes in both rods and cones, leading to a reduction in response sensitivity and an increase in response kinetics. However, there are distinct differences in the underlying mechanisms (Fu and Yau, 2007; Mustafi et al., 2009). For example, exposure of rods to steady, bright illumi-

\footnotetext{
Received Feb. 3, 2010; revised March 9, 2010; accepted March 27, 2010.

This work was supported by National Institutes of Health (NIH) Grants EY10336 (V.Y.A.), EY13729 (W.W.H.), EY11123 (W.W.H.), EY08571 (W.W.H.), and EY072224 (E.R.W.), NIH Core Grant for Vision Research EY5722 (V.Y.A. and E.R.W.), and grants from the Macular Vision Research Foundation, Foundation Fighting Blindness, Juvenile Diabetes Research Foundation, and Hope for Vision and Research to Prevent Blindness.

W.W.H. and the University of Florida have a financial interest in the use of AAV therapies, and own equity in a company (Applied Genetic Technologies Corporation) that might, in the future, commercialize some aspects of this work.

Correspondence should be addressed to Vadim Y. Arshavsky, Duke University Eye Center, 5012 AERI, 2351 Erwin Road, Durham, NC 27710. E-mail: vadim.arshavsky@duke.edu.

DOI:10.1523/JNEUROSCI.0613-10.2010

Copyright $\odot 2010$ the authors $\quad 0270-6474 / 10 / 306815-10 \$ 15.00 / 0$
}

nation causes a massive translocation of the G-protein transducin from the rod outer segment compartment, where visual signal transduction takes place, to other parts of the rod cell (Brann and Cohen, 1987; Philp et al., 1987; Whelan and McGinnis, 1988; Sokolov et al., 2002). Transducin is a key component of the phototransduction pathway, and the rate of transducin activation by the photoexcited visual pigment is a critical parameter determining the sensitivity of the photoresponse. Consequently, transducin translocation from rod outer segments causes a reduction in light sensitivity, which allows rods to operate under lighting conditions that would otherwise saturate their photoresponses (Sokolov et al., 2002). Cones naturally operate in brighter light than rods and would appear to benefit from such a mechanism even more than rods. Yet, practically all efforts to document transducin translocation in cones have failed (Elias et al., 2004; Kennedy et al., 2004; Coleman and Semple-Rowland, 2005; Rosenzweig et al., 2007); see, however, Chen et al., 2007). This suggests that the ability of cones to avoid response saturation in bright daylight is based on alternative molecular mechanisms.

In rods, transducin translocation requires its activation level to exceed a critical threshold (Lobanova et al., 2007), and we now demonstrate that the same principle applies to cones. Genetic manipulations increasing the rate of cone transducin activation or decreasing the rate of its inactivation allowed robust lightinduced translocation of both transducin subunits. However, we also show that the light intensity triggering cone transducin translocation in these animals completely suppressed the ability of cones to generate any further light responses. Therefore, our study reveals that the lack of transducin translocation in cones and their avoidance of "blinding" by bright light both rely on the same fundamental principle: the ability of cones to keep the amount of activated transducin below the translocation thresh- 
old by tightly controlling the activation/inactivation cycle of transducin under essentially any level of ambient illumination.

\section{Materials and Methods}

Antibodies. Anti-peptide antibodies against mouse $\mathrm{G} \alpha_{\mathrm{tc}}$ and $\mathrm{G} \gamma_{8}$ were generated in rabbits at the Duke University facility using the peptides CGIDYAEPSCADAGRQLNNL and CKEVKNPRDLISKTGVK, respectively, conjugated to KLH. The antibodies were affinity-purified from rabbit serum using the same peptides coupled to the Sulfolink Plus column (Pierce Biotechnology) according to the manufacturer's instructions. Note that the commonly used antibody against $\mathrm{G} \alpha_{\mathrm{tc}}$ (SC-390, Santa Cruz Biotechnology) is generated against the bovine protein and, when compared side by side, provided much weaker $\mathrm{G} \alpha_{\mathrm{tc}}$ immunostaining in mouse cones than our new antibody. Anti-PDE $\gamma_{c}$ antibodies were raised in rabbits against the peptide sequence CSDSPSLSPPAPSQ and affinity-purified as described above. Rabbit antibodies SC-389 against $\mathrm{G} \alpha_{\mathrm{t}}$ and goat antibodies sc-18413 against phosducin were purchased from Santa Cruz Biotechnology. Rabbit antibodies against rod PDE $\gamma$ were a gift from R.H. Cote (University of New Hampshire, Manchester, $\mathrm{NH})$. The secondary antibodies used for immunolocalization were Alexa Fluor 594 conjugated to goat anti-rabbit antibodies; the secondary antibodies used for Western blotting were Alexa Fluor 680 conjugated to goat or donkey anti-rabbit antibodies and donkey antigoat antibodies (Invitrogen).

Animal strains and procedures. C57BL/6 pigmented wild-type mice were purchased from Charles River Laboratories. $R 9 A P^{-1-}$ mice are described in Keresztes et al. (2004). RGS9 ${ }^{-1-}$ mice (Chen et al., 2000) and $G R K 1^{-1-}$ mice (Chen et al., 1999) were kindly provided by J.C.-K. Chen (Virginia Commonwealth University, Richmond, VA). $\mathrm{Nrl}^{-1-}$ mice (Mears et al., 2001) were a gift from A. Swaroop (National Eye Institute, Bethesda, MD). Mice were maintained under the standard 12/12 h light/dark cycle and dark adapted overnight before experiments, except for $G R K 1^{-I-}$ mice, which were raised in complete darkness to prevent photoreceptor degeneration. All light-exposure experiments were performed with animals anesthetized and their pupils dilated as described in Lobanova et al. (2007). Light $<100$ lux was delivered for $25 \mathrm{~min}$ from the source described in Sokolov et al. (2002); light of higher intensities was delivered for the same duration from the A20500 source (SCHOTT Lighting and Imaging) through fiber-optic light guides. The light intensity at the position corresponding to the mouse cornea surface was measured using a Traceable Dual-Range Light Meter (Fisher Scientific).

Immunolocalization of cone and rod transducin. Eyes were enucleated from dark- or light-adapted mice under an infrared microscope and fixed for 30 min with $4 \%$ paraformaldehyde in PBS buffer, $\mathrm{pH}$ 7.5. The anterior portions of the fixed eyes were removed under an infrared microscope, and the eyecups were further fixed overnight at $4^{\circ} \mathrm{C}$ wrapped with aluminum foil to prevent light exposure. Next day, the eyes were rinsed three times in PBS and embedded in $4 \%$ agarose (Invitrogen). One hundred-micrometer-thick cross sections from the central portion of the retina were collected with a vibratome (HM $650 \mathrm{~V}$, Microm) in 24 well plates, and floating sections were then incubated for $4 \mathrm{~h}$ with the blocking solution containing 3\% donkey serum and $0.1 \%$ Triton X-100 in PBS buffer on the orbital shaker. For detection of transducin subunits, sections were further incubated overnight with the corresponding rabbit antibodies, washed three times with PBS, incubated for $2 \mathrm{~h}$ with goat anti-rabbit Alexa Fluor 594 secondary antibody (1:500), washed three times in PBS, mounted with Fluoromount G (Electron Microscopy Sciences) under glass coverslips, and visualized using a Nikon Eclipse 90i confocal microscope.

Cloning, expression, and purification of recombinant proteins. Mouse $\mathrm{G} \alpha_{\mathrm{tc}}$ and $\mathrm{G} \gamma_{8} \mathrm{cDNA}$ clones were purchased from American Tissue Culture Collection. Since we have found that, as rod transducin, cone transducin could not be expressed in Escherichia coli, we constructed the chimeric cone $\mathrm{G} \alpha_{\mathrm{tc}} / \mathrm{G} \alpha_{\mathrm{i}}$ protein analogous to the rod transducin Chi6 described in Skiba et al. (1996) and subcloned it into the pet28b + vector between the NcoI and EcorI restriction sites. The coding sequence of cone $\mathrm{G} \alpha_{\mathrm{tc}} / \mathrm{G} \alpha_{\mathrm{i}}$ chimera was preceded by the MAHHHHHH tag for purification. The protein was expressed in E. coli and purified as described in Skiba et al. (1996). The chimeric protein had the same antibody recognition epitope as $\mathrm{G} \alpha_{\mathrm{tc}}$, which allowed us to use it as the standard for $\mathrm{G} \alpha_{\mathrm{tc}}$ quantification. The coding sequence of $\mathrm{G} \gamma_{8}$ lacking the last three amino acids (cleaved from the mature protein on its farnesylation in vivo) was subcloned into the pet $28 \mathrm{~b}+$ vector (Invitrogen) between NdeI and EcoRI sites. $\mathrm{G} \gamma_{8}$ expression in E. coli was induced for $4 \mathrm{~h}$ after induction with $0.8 \mathrm{~mm}$ isopropyl- $\beta$-D-1-thiogalactopyranoside for $4 \mathrm{~h}$. The protein was purified using Ni-NTA beads (Qiagen) followed by gel filtration on a Superose 12 column attached to the FPLC chromatography system (Pharmacia). Phosducin was expressed and quantified as described in Sokolov et al. (2004). Rod transducin subunits were prepared as described in Lobanova et al. (2008). Rod PDE $\gamma$ was a gift from Rick Cote (University of New Hampshire, Manchester, NH). The PDE $\gamma_{c}$ peptide was synthesized at Alpha Diagnostics International. The purity of all proteins was estimated to be at least $90 \%$. The concentrations of recombinant proteins were identified spectrophotometrically at $280 \mathrm{~nm}$ using the following extinction coefficients $\left(\right.$ all $\left.\mathrm{M}^{-1} \cdot \mathrm{cm}^{-1}\right)$ : $\mathrm{G} \gamma_{8}, 1490$; $\operatorname{PDE} \gamma, 6990 ; \operatorname{PDE} \gamma_{\mathrm{c}}, 5500$; phosducin, 16,530; G $\alpha_{\mathrm{t}}, 36,507 ; \mathrm{G} \alpha_{\mathrm{tc}} / \mathrm{G} \alpha_{\mathrm{i}}$ chimera, 42,207 (assuming that transducin contains a molecule of bound GDP); and $\mathrm{G} \beta_{1} \gamma_{1}, 58,410$.

Quantitative Western blotting. Protein quantification in rods of 60-dold wild-type mice was performed as described in Lobanova et al. (2008). For protein quantification in $\mathrm{Nrl}^{-1-}$ mouse retinas from 30-d-old animals (younger animals were used to avoid any complication from the retinal degeneration that occurs in $\mathrm{Nrl}^{-1-}$ mice at older ages) were harvested in $1.5 \mathrm{ml}$ Eppendorf tubes, frozen in liquid nitrogen, and solubilized in $250 \mu \mathrm{l}$ of $25 \mathrm{~mm}$ Tris- $\mathrm{HCl}, \mathrm{pH} 6.8$, containing 2\% SDS by rigorous sonication and vortexing. Total protein content in samples was measured using the RCDC Protein Assay Kit (Bio-Rad). Samples containing known amounts of total proteins were prepared by diluting them with the SDS-PAGE sample buffer, boiling them, and subjecting them to SDS-PAGE alongside standard samples containing known amounts of protein.

Preparation of $A A V$ and subretinal injections. The mouse rhodopsin cDNA was subcloned under cone preferential promoter PR2.1 (Li et al., 2008 ) in a standard adeno-associated virus (AAV) vector (pTR-PR2.1mRhodopsin). The vector was packaged in AAV serotype 5 virions and purified according to previously reported methods (Zolotukhin et al., 2002). A mixture of $0.5 \mu \mathrm{l}$ of AAV5-PR2.1-mRho and $0.5 \mu \mathrm{l}$ of AAV5PR2.1-GFP was coinjected subretinally in one eye of 3-week-old C57B/L mice. The vector titers of AAV5-PR2.1-mRho and AAV5-PR2.1-GFP were $4.3 \times 10^{13}$ and $8.4 \times 10^{12}$ virus genome $/ \mathrm{ml}$, respectively. The procedures for subretinal injections have been described previously (Timmers et al., 2001). Mice were killed 4 weeks after injection, and infected areas of their retinas were identified under a fluorescence microscope based on the green fluorescent protein (GFP) fluorescence signal and processed as above for $\mathrm{G} \alpha_{\mathrm{tc}}$ immunostaining.

Electroretinography. Electroretinography (ERG) signals were recorded using the Espion $\mathrm{E}^{2}$ system (Diagnosys) according to published methods (Saszik et al., 2002) essentially as described in Lobanova et al. (2008). Dark-adapted mice were anesthetized by intraperitoneal injection of a ketamine/xylazine mixture $(75 / 10 \mathrm{mg} / \mathrm{kg})$, their pupils were dilated with $1 \%$ cyclopentolate- $\mathrm{HCl}, 2.5 \%$ phenylephrine, and a drop of Gonak solution (Akorn) was placed on the cornea. The recording electrode was a silver fiber, and the reference electrode was a toothless alligator clip attached to the mouse cheek. Background illumination of 40 lux and test flashes producing $1000 \mathrm{~cd} \cdot \mathrm{s} / \mathrm{m}^{2}$ were generated by the Espion ColorDome ganzfeld illuminator (Diagnosys LLC). Background light ranging from 400 to 7000 lux was generated by the A20500 illuminator described above. Traces were processed with a $40 \mathrm{~Hz}$ high-frequency cutoff filter to remove oscillatory potentials. Animals were kept on a thermal blanket during the entire procedure.

\section{Results}

Why transducin does not translocate in cones: the hypothetical framework

To explain our experimental strategy for elucidating why transducin does not translocate in cones, we first have to introduce the underlying translocation mechanism in rods (for review, see 
Dim light

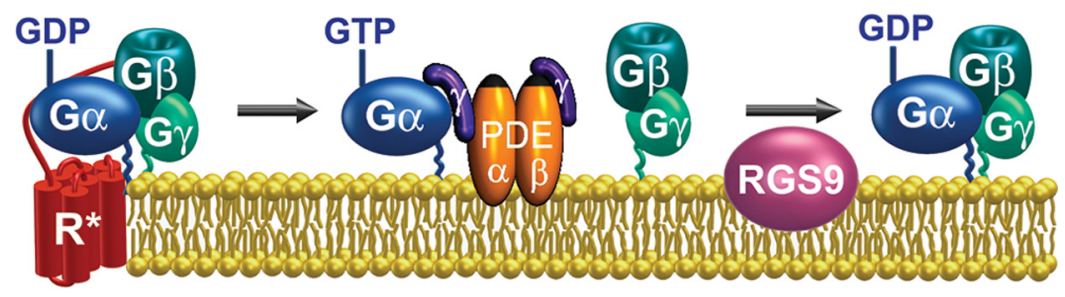

B

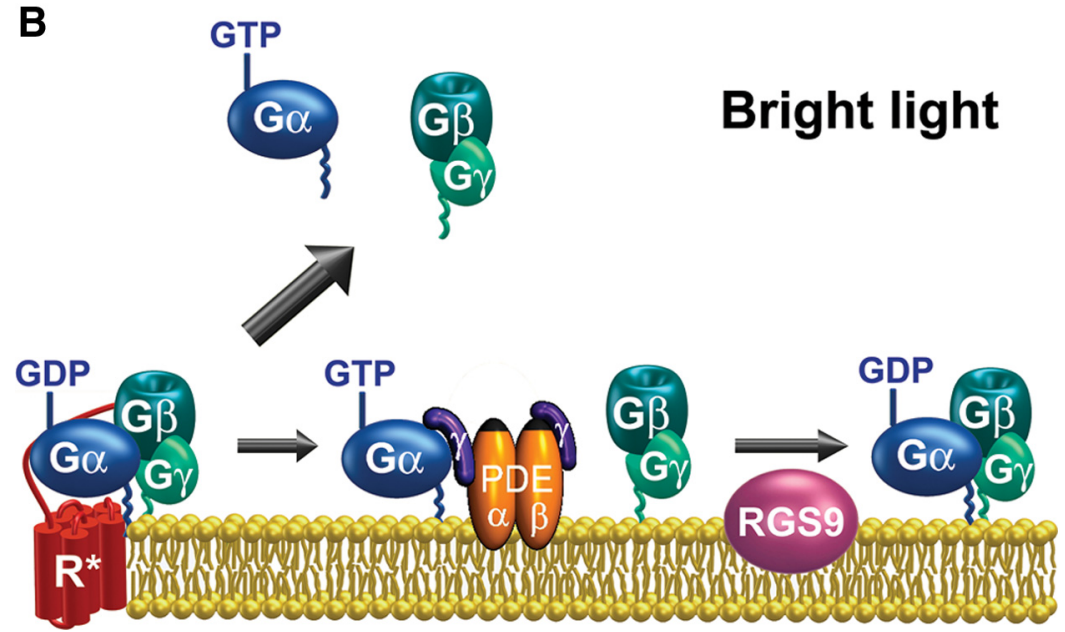

Figure 1. Diagram illustrating the light intensity threshold-dependent mechanism of transducin translocation. $\boldsymbol{A}$, At light intensities below the threshold, activated transducin binds to PDE and is rapidly inactivated by RGS9, providing little time for dissociation from the membrane. $\boldsymbol{B}$, At light intensities above threshold, more transducin is activated than can bind to PDE and be readily inactivated by RGS9. This led to a fraction of transducin staying activated sufficiently long enough to dissociate from the membrane to the cytosol. The amount of activated transducin in each case is represented by the thickness of the arrows. The basic elements of this diagram are reproduced with permission from Lobanova et al. (2007).

Burns and Arshavsky, 2005; Calvert et al., 2006; Fain, 2006; Artemyev, 2008; Slepak and Hurley, 2008). This mechanism is based on the difference in membrane affinities between the $\alpha \beta \gamma$ heterotrimer of transducin and its individual $\alpha$ - and $\beta \gamma$-subunits ( $\mathrm{G} \alpha_{\mathrm{t}}$ and $\mathrm{G} \beta_{1} \gamma_{1}$, respectively). As illustrated in Figure $1 A$, in dark-adapted rods the transducin heterotrimer is tightly associated with the membranes of photoreceptor outer segment discs due to the combined action of two lipid modifications: an acyl group on $\mathrm{G} \alpha_{\mathrm{t}}$ and a farnesyl group on $\mathrm{G} \gamma_{1}$. On transducin activation by photoexcited rhodopsin, $\mathrm{G} \alpha_{\mathrm{t}}$ binds GTP and separates from $G \beta_{1} \gamma_{1}$. Because each individual subunit has only one lipid modification, their membrane affinities become significantly reduced, allowing their dissociation from the disc membranes and subsequent diffusion from the outer segment through the rod cytoplasm (see Kosloff et al., 2008 for a recent detailed analysis of transducin interactions with membranes). However, this does not happen in light of dim-to-moderate intensity, in which the concentration of activated transducin does not exceed the concentration of its effector, cGMP phosphodiesterase (PDE). This is because PDE retains $\mathrm{G} \alpha_{\mathrm{t}}$ on the disc membranes via its own lipid modifications (Anant et al., 1992) and also serves as a cofactor for the GTPase activating protein RGS9 (Skiba et al., 2000), which rapidly inactivates $\mathrm{G} \alpha_{\mathrm{t}}$, leading to immediate restoration of transducin heterotrimer.

The situation changes dramatically in light of sufficient intensity to activate transducin in excess of PDE (Fig. $1 B$ ). The "additional" transducin can neither be retained on the discs by PDE nor be efficiently inactivated by RGS9. Consequently, the transducin subunits remain separated from PDE and from one another for a sufficient time to dissociate from the disc membranes and diffuse from the outer segment. This explains why transducin translocation in rods is triggered only when the intensity of steady illumination reaches a critical threshold level (Lobanova et al., 2007). Consistent with this framework, the threshold level can be reduced in mice having PDE replaced with a mutant that binds $\mathrm{G} \alpha_{\mathrm{t}} \cdot \mathrm{GTP}$ with reduced affinity (Lobanova et al., 2007). This level can also be reduced in mice lacking RGS9, in which transducin inactivates slower than normally, leading to an elevated steady-state transducin activation level (Kerov et al., 2005; Lobanova et al., 2007), or can be increased in mice overexpressing RGS9, thereby inactivating transducin at a faster rate (Lobanova et al., 2007).

Assuming that the same logic applies to cone-specific isoforms of the transducin subunits $\left(\mathrm{G} \alpha_{\mathrm{tc}}\right.$ and $\left.\mathrm{G} \beta_{3} \gamma_{8}\right)$, the inability of cone transducin to translocate even in very bright light suggests that its activation level never exceeds the critical level required for initiating translocation under any condition of steady illumination. This would be possible under two hypothetical scenarios. First, it is conceivable that cones contain much more PDE than rods, so that a larger number of activated transducin molecules could be retained on the discs by PDE. Second, it is possible that cones exert a much tighter control of the transducin activation/inactivation cycle than rods, so that the concentration of activated transducin cannot exceed that of PDE more than momentarily. Both hypotheses were evaluated in our study.

\section{Rods and cones have comparable molar ratios of PDE and transducin}

In the first set of experiments, we explored the relative expression levels of transducin and PDE in mouse cones. The low abundance of cones in normal mouse retinas makes quantification of conespecific proteins very difficult. Therefore, we conducted these measurements in mice lacking the transcription factor $\mathrm{Nrl}$, in which all photoreceptors closely resemble S-cones (Mears et al., 2001; Daniele et al., 2005; Nikonov et al., 2005). Most importantly, these cone-like photoreceptors have three hallmark properties of cones that make them an adequate model for studying cone transducin translocation mechanisms. They express cone isoforms of visual pigments, transducin and phosphodiesterase, display cone-like parameters of photoresponses, and, like cones, cannot be blinded by bright light and lack transducin translocation under any conditions of illumination. The latter was thoroughly documented by Rosenzweig et al. (2007), who used a broad range of illumination conditions including those photoexciting $>70 \%$ of the visual pigment. The molar ratios among cone transducin $\alpha$-subunit $\left(\mathrm{G} \alpha_{\mathrm{tc}}\right)$, cone transducin $\gamma$-subunit $\left(\mathrm{G} \gamma_{8}\right)$, and cone PDE $\gamma$-subunit $\left(\operatorname{PDE} \gamma_{\mathrm{c}}\right)$ in $\mathrm{Nrl}^{-1-}$ retina lysates was 
determined by quantitative Western blotting using the corresponding recombinant proteins as standards (Fig. $2 A-C$ ). We also measured the amount of phosducin, the photoreceptor-specific protein previously shown to assist the light-driven translocation of G $\beta_{1} \gamma_{1}$ in rods (Sokolov et al., 2004) (Fig. 2D). The data averaged from six experiments indicated that the molar ratios among these four proteins are (mean $\pm \mathrm{SD}): \operatorname{PDE} \gamma_{\mathrm{c}}, 1 ; \mathrm{G} \alpha_{\mathrm{tc}}, 6.0 \pm$ 0.3 ; $\mathrm{G} \gamma_{8}, 4.0 \pm 0.4$; phosducin, $5.7 \pm 0.1$. For consistency, we applied the same quantification protocol to measure the ratios among the corresponding rodspecific proteins in wild-type mice (supplemental Fig. 1, available at www.jneurosci. org as supplemental material) and found them to be as follows (mean $\pm \mathrm{SD} ; n=2$ ): $\operatorname{PDE} \gamma, 1 ; \mathrm{G} \alpha, 9.2 \pm 0.4 ; \mathrm{G} \gamma_{1}, 9.3 \pm 0.6$; phosducin, $8.8 \pm 0.4$. These are within the range of several previous quantifications in the mouse (Sokolov et al., 2004; Krispel et al., 2007; Lobanova et al., 2008).

These measurements indicate that the molar ratio between PDE and transducin $\alpha$-subunit in $\mathrm{Nrl}^{-1-}$ cones is only $\sim 1.5$ fold higher than the corresponding ratio in rods and that, just like rods, cones contain approximately equimolar amounts of transducin and phosducin. This is generally consistent with a previous report that the molar ratio between cone PDE and visual pigment in the cone-rich chipmunk retina is comparable to the corresponding ratio of the rod proteins in the mouse and bovine retinas (Zhang et al., 2003). Therefore, cones contain significantly more transducin than PDE, and the lack of cone transducin translocation cannot be explained by its complete retention in a stoichiometric complex with PDE.

\section{Knockout of the GTPase activating complex enables transducin translocation in cones}

We next tested our second hypothesis that cone transducin does not translocate because its activated, GTP-bound fraction remains below the critical threshold level under all conditions of steady illumination. The amount of activated transducin is set by the rate of its activation by photoexcited visual pigment and the rate of its inactivation through GTP hydrolysis; each of these rates might be different in rods and cones.

We first evaluated the impact of transducin's inactivation rate, inspired by reports that cones express more RGS9 than rods (Cowan et al., 1998) and that, in rods, the knockout of RGS9 lowers the threshold light intensity required for initiating transducin translocation (Kerov et al., 2005; Lobanova et al., 2007). We analyzed transducin translocation in cones of the R9AP knock-out mouse, which lacks all three proteins forming the GTPase activating complex for transducin: RGS9, G $\beta 5$, and R9AP (Keresztes et al., 2004). In striking contrast to wild-type cones, $R 9 A P^{-1-}$ cones displayed significant translocation of both transducin subunits (Fig. 3). Strong $\mathrm{G} \alpha_{\mathrm{tc}}$ and $\mathrm{G} \gamma_{8}$ immunostaining was detected in cone inner segments, around the nuclei and at the

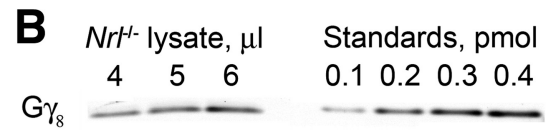

Standards, pmol $\begin{array}{llll}0.1 & 0.2 & 0.3 & 0.4\end{array}$

$\mathrm{G} \gamma$
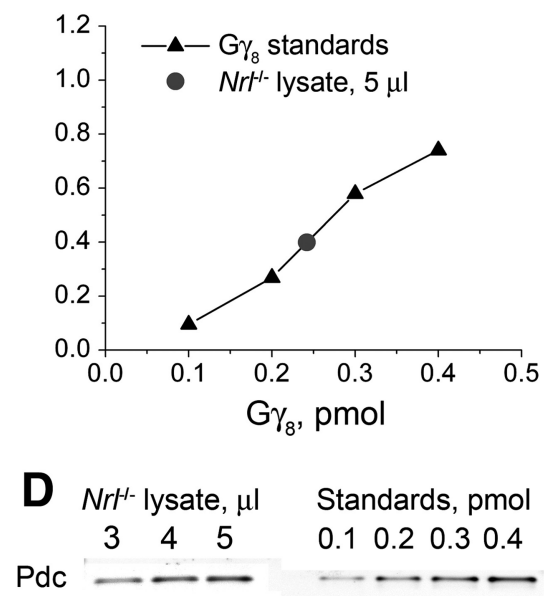

Standards, pmol $\begin{array}{lllllll}0.1 & 0.2 & 0.3 & 0.4\end{array}$

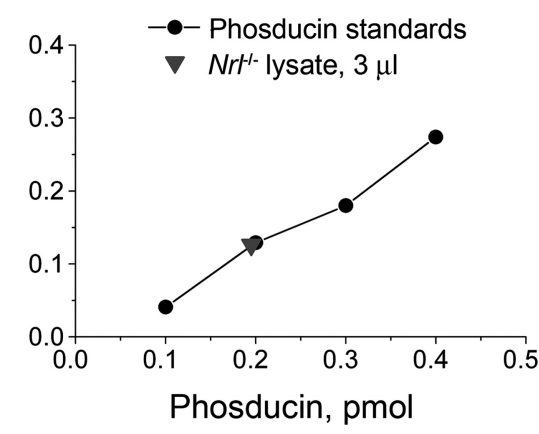

Figure 2. $\quad \boldsymbol{A}-\boldsymbol{D}$, Determination of the molar ratio among transducin subunits $\left(\boldsymbol{A}, \mathrm{G} \alpha_{\mathrm{tc}} ; \boldsymbol{B}, \mathrm{G} \gamma_{8}\right), \operatorname{PDE} \gamma_{\mathrm{c}}(\boldsymbol{C})$, and phosducin (Pdc; $D)$ in cones of $\mathrm{Nrl}^{-1-}$ mice. Retina lysate aliquots of indicated volumes were separated by SDS-PAGE along with $0.1,0.2,0.3$, and each protein are shown below the blots. All data were obtained with retina extract obtained from a single animal; the results obtained in multiple experiments are summarized in the text.

synaptic terminals. These data provide the first demonstration that transducin can translocate in physiologically intact mouse cones and establish that transducin's inactivation rate serves as a critical factor precluding its translocation in wild-type mouse cones.

As in rods, transducin translocation in $R 9 A P^{-1-}$ cones was observed only when the intensity of steady illumination exceeded a critical level, with the first signs of translocation observed in the light intensity range of 2000-4000 lux at the cornea surface, slightly varying among individual animals (Fig. 4, upper panel; see also Fig. $7 B$ ). This result explains why Rosenzweig et al. (2007), who used a single light intensity of 500 lux, did not observe cone transducin translocation in an essentially identical model, the RGS9 knock-out mouse. Our own control experiments with $R G S 9^{-1-}$ mice demonstrated that robust $\mathrm{G} \alpha_{\mathrm{tc}}$ translocation from cone outer segments can be induced by sufficiently bright light (supplemental Fig. 2, available at www.jneurosci.org as supplemental material).

Importantly, the light intensity threshold for transducin translocation in $R 9 A P^{-1-}$ cones was $\sim 1000$-fold higher than that required to initiate transducin translocation in rods of the same mice (Fig. 4, bottom). Considering that the absence of RGS9 similarly slows the inactivation rates of the rod and cone responses (Lyubarsky et al., 2001), this result suggests that to achieve concentrations of activated transducin comparable to PDE in cones and rods, it is necessary to photoexcite substantially 


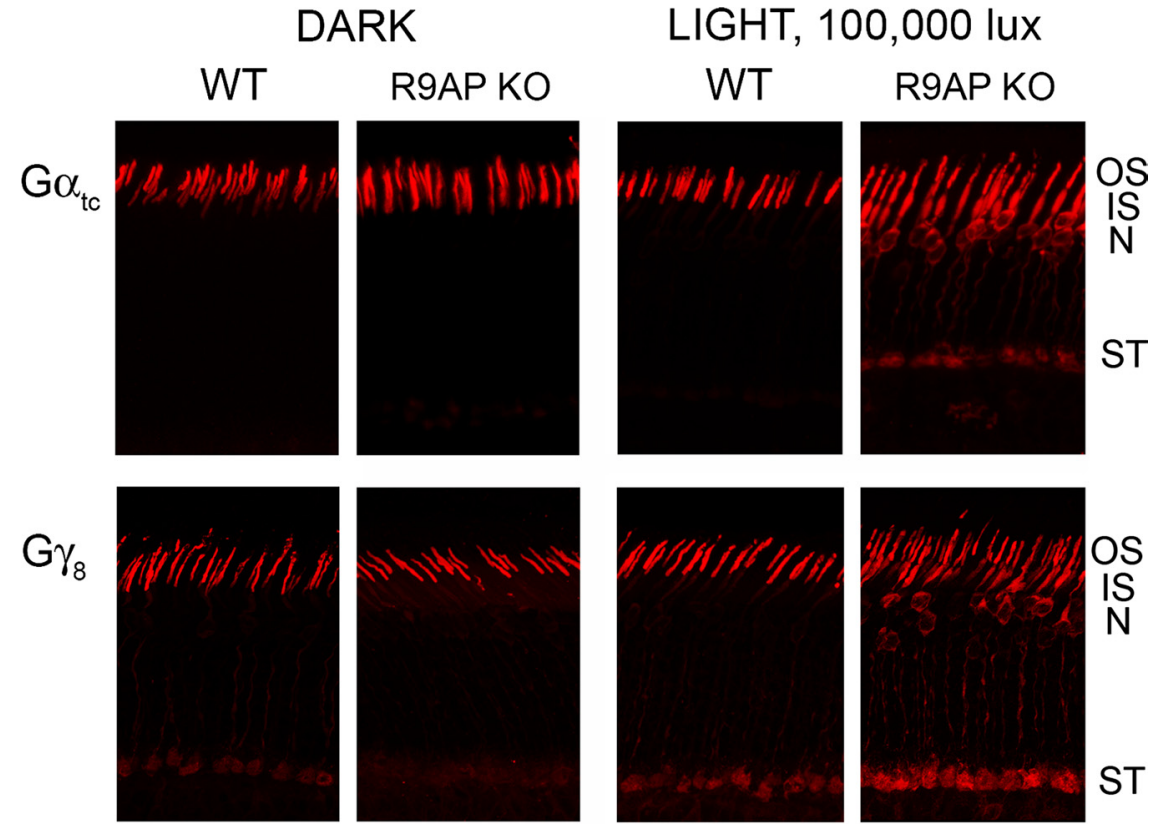

Figure 3. Transducin translocation in cones of R9AP knock-out mice. Wild-type $C 57 \mathrm{BL} / 6$ or $R 9 A P^{-1-}$ mice were either dark adapted or exposed to 25 min of illumination from a calibrated light source producing a light intensity of 100,000 lux at the cornea surface. Animals were killed, eyes fixed, and retina cross sections stained with antibodies against $\mathrm{G} \alpha_{\mathrm{tc}}$ (top panels) or $\mathrm{G} \gamma_{8}$ (bottom panels). Note that weak $G \gamma_{8}$ immunostaining was observed in cone synaptic terminals of wild-type and dark-adapted $R 9 A P^{-1-}$ mice, perhaps due to its engagement into $\mathrm{G} \beta \gamma$ complexes distinct from transducin. However, this signal was further enhanced in light-adapted $R 9 \mathrm{AP}^{-1-}$ cones. The subcellular compartments of the cone are labeled as follows: 0S, Outer segment; IS, inner segment; N, nucleus; ST, synaptic terminal.

more cone than rod pigment. Since electrophysiological studies indicate that the initial rates of transducin activation by rod and cone pigments in the mouse are not more than approximately twofold different (Nikonov et al., 2006) (see Discussion for more detail), this led us to suggest that the vast difference in transducin translocation thresholds is explained by a much shorter lifetime of the cone visual pigment in its photoexcited state. We tested this hypothesis in the next series of experiments.

\section{Knockout of rhodopsin kinase affects transducin translocation in rods but not in cones}

Although the photoexcited intermediates of both the rod and cone visual pigments $\left(\mathrm{R}_{\mathrm{r}}{ }^{*}\right.$ and $\mathrm{R}_{\mathrm{c}}{ }^{\star}$, respectively) are able to inactivate themselves through thermal decay, the rate of inactivation can be enhanced by a two-step biochemical mechanism consisting of pigment phosphorylation by rhodopsin kinase and the subsequent binding of arrestin, which completely blocks the pigments' ability to activate transducin (for review, see Arshavsky, 2002; Gurevich and Gurevich, 2003; Maeda et al., 2003). In the mouse, rods and cones express the same rhodopsin kinase isoform, GRK1 (Weiss et al., 2001), which enabled us to analyze the impact of this mechanism on transducin translocation in rods and cones using the same GRK1 knock-out mouse model.

The data presented in Figure $5 A, B$ demonstrate that rod transducin translocation in $G R K 1^{-1-}$ animals is initiated at approximately twofold to threefold dimmer light than in wildtype mice. This is similar to the effect caused by the knockout of R9AP (Lobanova et al., 2007) and suggests that each of the phototransduction inactivation mechanisms $-\mathrm{R}_{\mathrm{r}}{ }^{*}$ phosphorylation by GRK1 and transducin GTPase acceleration by RGS9 - make comparable contributions to setting the steadystate level of activated transducin in rods subjected to bright, steady illumination.
In contrast, the knockout of GRK1 did not allow transducin translocation in cones under a broad range of steady illumination, including $\sim 20$-fold brighter light than that required to initiate transducin translocation in $R 9 A P^{-1-}$ cones (Fig. $5 C$ ). This observation indicates that the "classic" GRK1/arrestin-dependent mechanism of $\mathrm{R}_{\mathrm{c}}{ }^{*}$ inactivation plays a lesser role than transducin inactivation by RGS9 in setting the steady-state level of activated transducin in cones exposed to bright light. It further suggests that the rod/cone differences with respect to transducin translocation may originate from other properties of their photoexcited visual pigments, for example, the different rates of their spontaneous thermal decay (Shichida et al., 1994; Imai et al., 1997).

Light-driven transducin translocation in cones can be induced by expression of rod visual pigment

To evaluate whether the rod/cone difference in regard to transducin translocation is largely explained by the intrinsic molecular properties of their visual pigments, we tested whether transducin translocation in cones can be triggered by the expression of rod pigment (rhodopsin). This approach was possible because previous studies with genetically manipulated animals demonstrated that rod pigment can activate cone transducin and cone pigment can activate rod transducin in intact photoreceptors (Kefalov et al., 2003; Rosenzweig et al., 2007; Sakurai et al., 2007; Shi et al., 2007; Deng et al., 2009). To express rhodopsin in cones, we took advantage of the recently developed technique of adeno-associated, virus-mediated gene delivery to the mouse retina and driving expression with the cone opsinspecific promoter (Alexander et al., 2007). Three-week-old wildtype mice were infected by subretinal virus injections, and transducin translocation in their cones was analyzed 4 weeks later. Representative images in Figure 6 indicate that light exposure caused efficient transducin translocation in the majority of cones from the infected region of these animals' retinas. The lowest light intensity sufficient to induce this effect was 200 lux, which is $10-20$-fold lower than for $R 9 A P^{-1-}$ mice. These data indicate that even in cones in which both rod and cone visual pigments coexist, the critical level of transducin activation required for translocation is achieved at much dimmer illumination than in cones in which transducin inactivation is slowed. Therefore, the differences in the intrinsic properties of rod and cone visual pigments indeed serve as the most important factor allowing transducin translocation in rods and preventing it in cones.

Light intensities required for cone transducin translocation in $R_{9 A P^{-/-}}$mice saturate cone light responses

Elucidating the conditions required to induce transducin translocation in cones allowed us to directly evaluate another key prediction of the mechanism illustrated in Figure 1. If transducin translocation is indeed triggered just as its activation level exceeds that of PDE, then a background light intensity at or above the translocation threshold is predicted to make cones insensitive to 


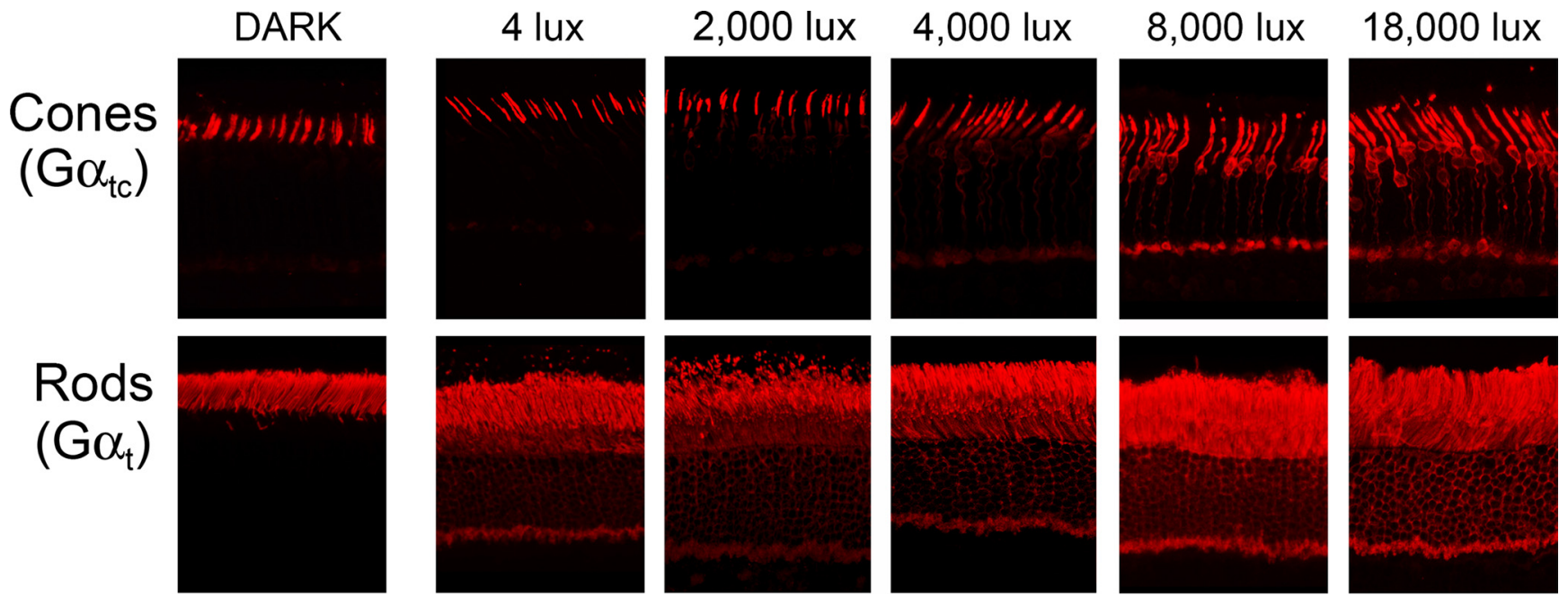

Figure 4. The light-dependency of cone transducin translocation in R9AP knock-out mice. The animals were treated as described in the Figure 3 legend at the light intensities indicated above each panel. Retina cross sections were immunostained with antibodies against $\mathrm{G} \alpha_{\mathrm{tc}}$ (top panels) or $\mathrm{G} \alpha_{\mathrm{t}}$ (bottom panels).

any further light stimuli. This is because all available PDE molecules are already activated by transducin: any further increases in the level of photoexcited visual pigment therefore could not be translated into changes in PDE activity, cGMP concentration, and channel permeability. We tested this prediction in $R 9 A P^{-1-}$ mice using ERG, which is a noninvasive electrophysiological technique that allows monitoring of the light-evoked activity of retinal neurons by an electrode positioned at the corneal surface (Saszik et al., 2002). An ERG signal consists of an initial negative deflection (a-wave) originating primarily from the light-dependent suppression of the circulating current in photoreceptor outer segments and a subsequent positive deflection (b-wave) reflecting the amplified response of the downstream ONbipolar cells. In a typical experiment (Fig. 7), we recorded ERGs from an anesthetized mouse subjected first to 40 lux illumination completely saturating its rods (Lyubarsky et al., 1999), then to a step of brighter light either below or above the transducin translocation threshold, and finally to 40 lux again. Control experiments conducted with wild-type mice revealed the presence of distinct conedriven ERG responses at all tested light intensities (Fig. $7 B, C$ ). ERGs from $R 9 A P^{-1-}$ mice were very similar both at 40 lux (although signals recovered somewhat slower, perhaps reflecting slower recovery of photoresponses) and up until the light intensity reached a level of 2000 lux, at which point they became completely unresponsive to light. Control immunostaining of retinas collected immediately after the last ERG recording (Fig. $7 B$, side panels) demonstrated that first signs of transducin translocation were evident at this very light intensity. These results provide striking evidence that the level of transducin activation required for its transloca-

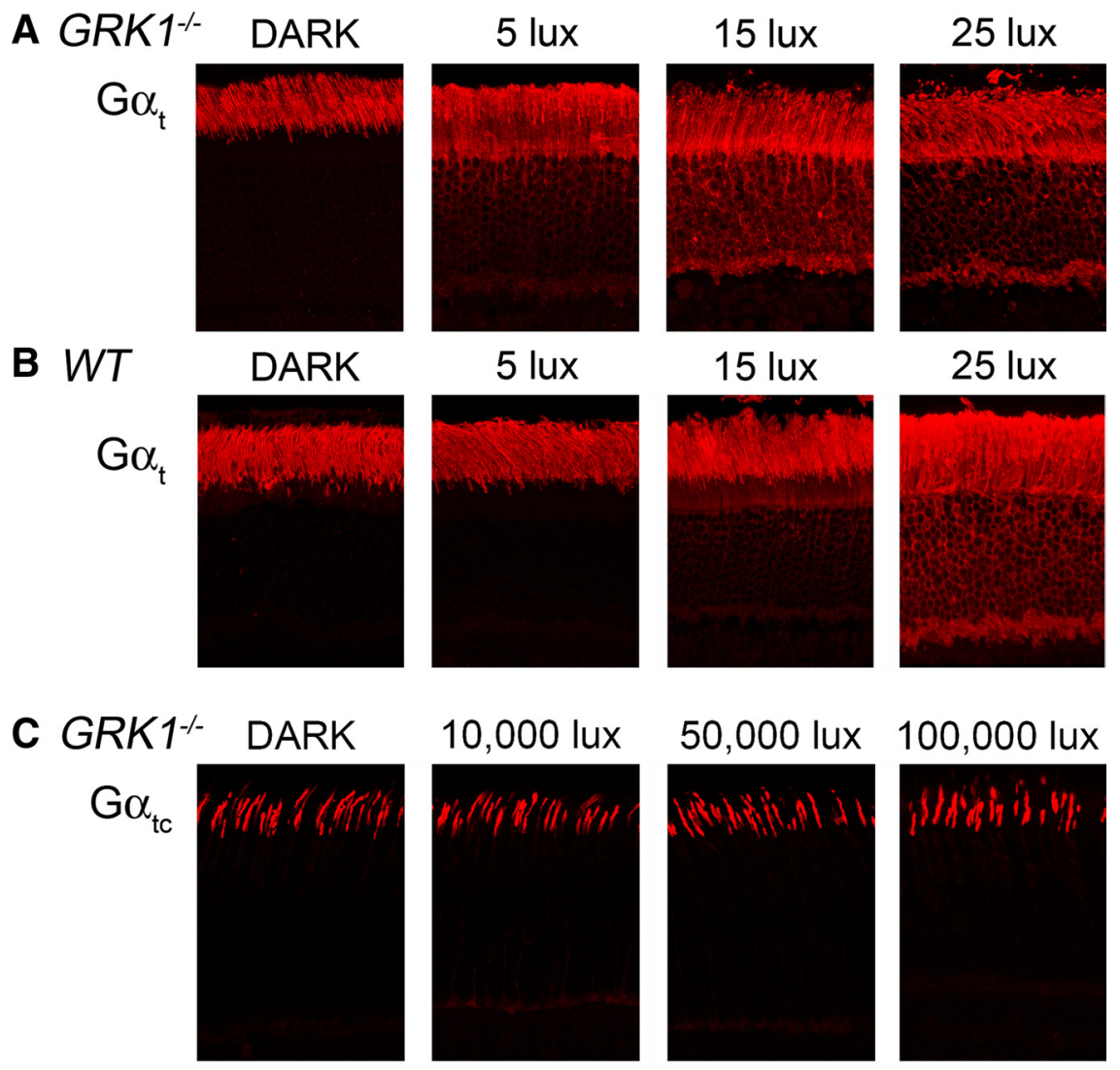

Figure 5. Transducin translocation in rods and cones of GRK1 knock-out mice. $A, B, G R K 1^{-1-}(A)$ and wild-type mice $(\boldsymbol{B})$ were either kept in the dark or exposed to 25 min illumination from a calibrated light source producing the light intensities indicated above the panels. Animals were killed and retina cross sections were stained with antibodies against $\mathrm{G} \alpha_{\mathrm{t}}$. C, A similar protocol using brighter illumination levels was applied to $G R K 1^{-1-}$ mice, and the sections were stained with antibodies against $\mathrm{G} \alpha_{\mathrm{tc}}$.

tion indeed saturates the biochemical phototransduction cascade and renders these cells insensitive to light.

The blinding effect of bright light on $R 9 A P^{-1-}$ cones was reversible since cone-driven ERGs recorded from these animals after returning to the 40 lux background light were comparable to those recorded from wild-type mice (Fig. $7 B$, lower traces). Under this condition, both animal types displayed smaller responses 


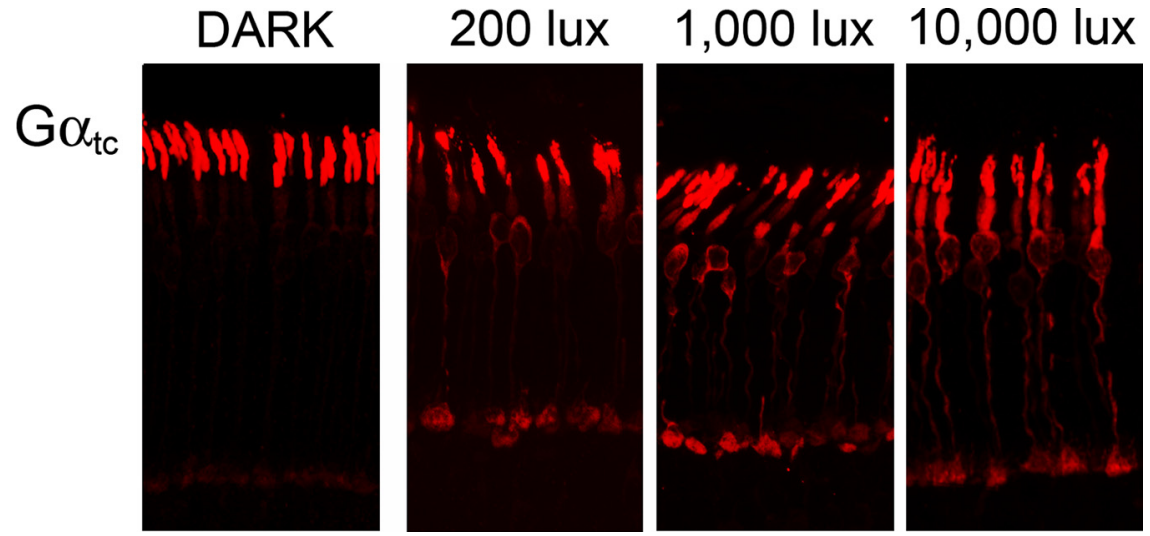

Figure 6. Cone transducin translocation in cones expressing rod visual pigment. Representative $\mathrm{G} \alpha$ ic immunostaining in retina sections obtained from wild-type mice coinfected with AAV5-PR2.1-mRhodopsin and AAV5-PR2.1-GFP. A total of 13 animals were tested in the dark or at a range of light intensities from 10 to 100,000 lux. Animals were killed and their eyes fixed. Retina cross sections were obtained from portions of the retina displaying the brightest GFP fluorescence and stained with antibodies against $\mathrm{G} \alpha_{\mathrm{tc}}$.

than those recorded during the initial 40 lux exposure, which is likely to be explained by bright light bleaching significant fractions of their cone pigments and/or evoking long-lasting adaptive effects in the retina. It is also worth noting that our previous study demonstrated that rods that have recovered from saturating illumination causing transducin translocation produce less sensitive ERG a-waves than they did before illumination, which we interpreted as a reflection of the decreased transducin activation rate caused by translocation (Sokolov et al., 2002). We then observed that the cone a-wave restoration in $\mathrm{R} \mathrm{AP}^{-1-}$ mice was less pronounced than in wild-type mice in essentially all animals subjected to the brightest light condition. However, we could not analyze this effect quantitatively due to very small size of these signals and the fact that, unlike rod a-waves, cone a-waves originate from both cones and the downstream OFF-bipolar cells (Sharma et al., 2005).

\section{Discussion}

Rods and cones have distinctly different roles in the visual system. While rods are primarily responsible for operating under limited lighting conditions, cones take over in daylight and provide better temporal and spatial resolution than rods. Yet the entire retina becomes exposed to bright light during the daytime, and both cell types must adjust their biochemical behavior to cope with this condition. One challenge they face is to efficiently control very large amounts of activated transducin. Photoreceptors express more G-protein than any other cells, which allows them to maximize the rate of transducin activation by photoexcited visual pigments and produce reliable responses in dim light (for review, see Arshavsky et al., 2002). However, high gain of transducin activation presents a challenge in bright light when large amounts of activated transducins could easily saturate the photoresponse. Rods and cones approach this challenge differently. In rods, the gain of transducin activation can be reduced via light-driven transducin translocation, which takes place when the amount of activated transducin in rods exceeds a critical level. We now demonstrate that genetic manipulations increasing the level of activated transducin in cones allow vigorous translocation as well, but these conditions also render cones insensitive to light. This situation is avoided in wild-type cones through an extremely rigid control of transducin's activation/inactivation cycle by its upstream activator, the photoexcited pigment, and its downstream inactivator, RGS9.
The central role of rapid $R_{c}^{*}$ inactivation in preventing transducin translocation in cones

Although we were able to induce robust transducin translocation in mouse cones either by boosting transducin activation (via expression of rod visual pigment) or by slowing its inactivation (via knocking out the R9AP or RGS9 genes), the former produced a much stronger effect, inducing translocation at $\sim 10$-fold dimmer light ( $\sim 200$ vs $\sim 2000$ lux $)$. In these experiments, the amounts of rhodopsin expressed in cones varied from cell to cell and were unlikely to have ever reached the expression level of normal cone pigment. Therefore, the fact that even fractional expression of rhodopsin in cones allows translocation at $\sim 200$ lux predicts that full levels of rhodopsin expression would trigger transducin translocation at a light intensity even closer to the $8-10$ lux range of wild-type rods. This suggests that the rod/cone differences in transducin translocation can be attributed primarily to specific differences in the properties of their visual pigments.

There are three non-mutually exclusive theoretical mechanisms that could explain why rhodopsin expression in cones makes transducin translocation possible: (1) more efficient pigment photoexcitation by white light; (2) a higher rate of transducin activation; and (3) a longer lifetime of the photoactivated pigment intermediate $\mathrm{R}_{\mathrm{r}}{ }^{*}$. The first is likely to be insignificant for the majority of mouse cones that express the M-cone opsin, the absorbance spectrum of which is only $\sim 10 \mathrm{~nm}$ different from that of rhodopsin (Yokoyama and Yokoyama, 2000). The other two mechanisms are supported by many published studies.

Although both biochemical and electrophysiological studies in fish suggest that $\mathrm{R}_{\mathrm{c}}{ }^{*}$ activates transducin at a significantly lower rate than $\mathrm{R}_{\mathrm{r}}^{*}$ (Tachibanaki et al., 2001; Kawamura and Tachibanaki, 2008), this difference is much smaller in mammals. Comparative measurements of signal amplification by single-cell recordings indicated that the rate of transducin activation in mouse cones is only approximately twofold slower than in rods (Nikonov et al., 2006). This leads us to conclude that the key property of rhodopsin that drove transducin translocation in mouse cones is the long lifetime of its $\mathrm{R}_{\mathrm{r}}^{*}$ state.

A photoexcited pigment can be inactivated through either thermal decay or the biochemical mechanism of phosphorylation/arrestin binding. The fact that the thermal decay of $\mathrm{R}_{\mathrm{c}}{ }^{*}$ is much more rapid than that of $\mathrm{R}_{\mathrm{r}}^{*}$ is well documented in spectroscopic studies (Shichida et al., 1994; Imai et al., 1997). Single-cell recordings further strengthened this conclusion by demonstrating that the delay in photoresponse recovery caused by the knockout of GRK1 and/or arrestin(s) is strikingly more significant in rods than in cones, indicating that the spontaneous decay rate of $\mathrm{R}_{\mathrm{c}}{ }^{*}$ is much high than that of $\mathrm{R}_{\mathrm{r}}^{*}$ (Xu et al., 1997; Chen et al., 1999; Lyubarsky et al., 2000; Nikonov et al., 2005, 2008). For example, arrestin-independent photoresponse inactivation is 70fold more rapid in cones than in rods (Nikonov et al., 2008). Pigment phosphorylation was also shown to occur much faster in cones than in rods, at least in the fish species where a direct comparison was performed (Tachibanaki et al., 2001, 2005; Kennedy et al., 2004). Therefore, our conclusion that the lack of transducin translocation in cones is explained primarily by a 
much shorter lifetime of $\mathrm{R}_{\mathrm{c}}^{*}$ than $\mathrm{R}_{\mathrm{r}}^{*}$ is well consistent with all available data. But how to reconcile this conclusion with our new result that the knockout of GRK1 promotes transducin translocation in rods but not cones?

Multiple biochemical studies demonstrated that, both in rods and cones, rhodopsin kinase can be easily saturated by its substrate, $\mathrm{R}^{\star}$, and the phosphorylation rate of individual $\mathrm{R}^{\star}$ molecules falls drastically as the light intensity approaches the levels required for transducin translocation (e.g., Sitaramayya and Liebman, 1983; Binder et al., 1990; Kennedy et al., 2001; Kennedy et al., 2004). Therefore, our data do not contradict the critical role of $\mathrm{R}_{\mathrm{c}}{ }^{*}$ inactivation through phosphorylation/arrestin binding in shaping cone photoresponses to dim-to-moderate light (Nikonov et al., 2005, 2008). Rather, they suggest that the balance between this classic inactivation mechanism and pigment thermal decay shifts toward the latter on an increase in illumination intensity. Accordingly, the steady-state level of activated transducin in bright light is critically dependent on the thermal decay rate of $\mathrm{R}^{\star}$, which is much more rapid in cones.

\section{Other factors potentially affecting} transducin translocation in cones The critical role of $\mathrm{R}_{\mathrm{c}}{ }^{\star}$ lifetime in precluding cone transducin translocation does not exclude additional contributions from other mechanisms. One is the faster rate of transducin inactivation in cones than in rods. Single-cell recordings indicate that cones recover from excitation twofold to threefold faster than rods (Nikonov et al., 2006), and biochemical estimates show that cones contain approximately fourfold more RGS9 [1:62 molar ratio to cone opsin in the chipmunk (Zhang et al., 2003) vs 1:269 ratio to rhodopsin in the mouse (Martemyanov et al., 2008)]. Another factor may be the $\sim 1.5$-fold higher molar ratio between PDE and transducin documented in this study, which would allow cones to activate $\sim 50 \%$ more transducin until a fraction of activated $\alpha$-subunit would exceed the capacity of PDE to retain it on the disc membranes. Finally, transducin translocation in cones may be impeded by the nature of $\mathrm{G} \alpha_{\mathrm{tc}}$ lipid modification. While the entire pool of $\mathrm{G} \alpha_{\mathrm{tc}}$ is myristoylated (Rosenzweig et al., 2007), up to $\sim 90 \%$ of $\mathrm{G} \alpha_{\mathrm{t}}$ in rods is modified by less lipophilic acyl groups (Neubert et al., 1992). Myristoylation of $\mathrm{G} \alpha_{\mathrm{t}}$ or $\mathrm{G} \alpha_{\mathrm{tc}}$ does not block their ability to translocate (Rosenzweig et al., 2007), yet myristoylated $\mathrm{G} \alpha_{\mathrm{t}}$ in rods translocates less efficiently than $\mathrm{G} \alpha_{\mathrm{t}}$ modified by less lipophilic species (Lobanova et al., 2007).

On the other hand, it is unlikely that cone transducin does not translocate because its subunits do not separate on activation (Rosenzweig et al., 2007), a hypothesis adopted after not detecting transducin translocation in $R G S 9^{-1-}$ cones. We now demon-

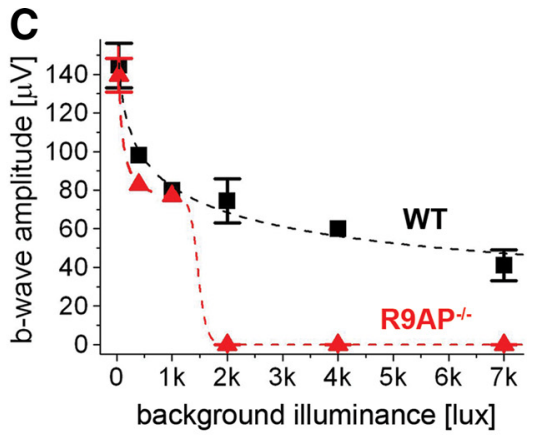

R9AP-1-
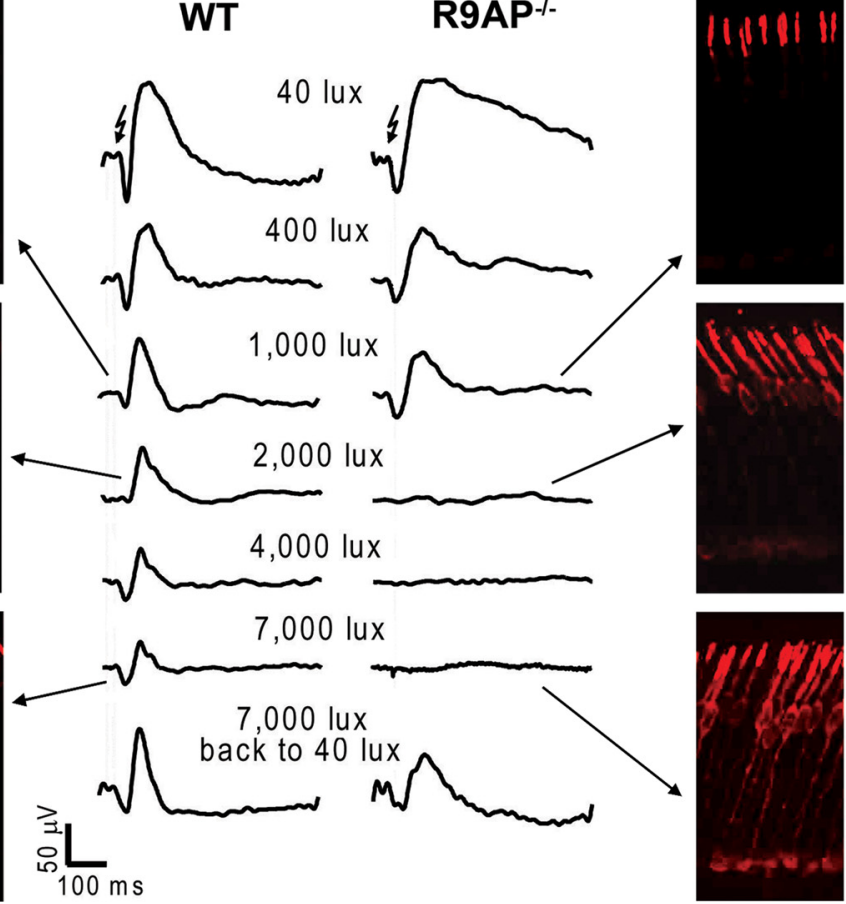

Figure 7. Evaluation of cone light responses at background light below and above transducin translocation threshold in R9AP

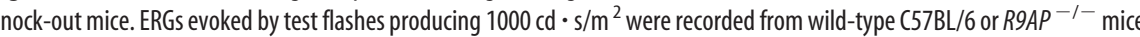
cotely suppress rod-driven responses, next after applying bright light of the various intensities indicated for $25 \mathrm{~min}$, and after returning to 40 lux illumination for a period of $6 \mathrm{~min}$ (first flash applied $1 \mathrm{~min}$ after cessation of bright light). The period veraged from 6 ( 40 lux) or 25 (other conditions) individual trials are shown in the middle. Representative immunostaining images of retina cross sections from selected illumination conditions (marked by arrows) are shown on the sides. $C$, The averaged amplitudes of ERG b-waves recorded under the initial 40 lux condition and all conditions of bright illumination are plotted as a function of background illuminance. Data points are connected by smooth dashed lines.

strate that cone transducin vigorously translocates in $R G S 9^{-1-}$ mice exposed to light of sufficient intensity, as well as in cones expressing the rod visual pigment. Because neither genetic manipulation affected cone transducin directly, the inability of transducin to translocate in wild-type cones can be sufficiently explained by the specific parameters of its activation/inactivation cycle rather than the separation pattern of its subunits.

\section{Physiological advantages and disadvantages of transducin translocation as a light adaptation mechanism}

The fact that transducin translocates in wild-type rods but not cones is not immediately intuitive. Rods adopt this mechanism for extending the range of their light responsiveness and may also benefit from preventing excessive energy consumption by the phototransduction cascade when they contribute little to vision 
(for review, see Burns and Arshavsky, 2005; Calvert et al., 2006; Fain, 2006; Artemyev, 2008; Slepak and Hurley, 2008). Cones customarily operate in brighter light than rods and would appear to benefit even more from adjusting signal amplification of their photoresponses under these conditions. However, we now demonstrate that cone transducin only translocates under conditions blinding the cell. Thus, to perform translocation, a cone has to remain unresponsive to light for the many minutes required for transducin to move away from the outer segment. Clearly, such a condition is not compatible with physiological behavior of normal cones, which are never blinded by light and completely adapt to ambient illumination of the brightest intensity within a period of only a few seconds (Rodieck, 1998). Rods, on the other hand, contribute little to daytime vision, and their saturation by bright light, required for transducin translocation, does not impair the overall visual function of a typical vertebrate animal. Thus, the presence or absence of transducin translocation can be viewed as an evolutionary adaptation of each photoreceptor type: translocation allows rods to transition into a deeply light-adapted, energy-saving mode, while its absence in cones enables them to avoid blindness under all natural conditions.

\section{References}

Alexander JJ, Umino Y, Everhart D, Chang B, Min SH, Li Q, Timmers AM, Hawes NL, Pang JJ, Barlow RB, Hauswirth WW (2007) Restoration of cone vision in a mouse model of achromatopsia. Nat Med 13:685-687.

Anant JS, Ong OC, Xie HY, Clarke S, O’Brien PJ, Fung BK (1992) In vivo differential prenylation of retinal cyclic GMP phosphodiesterase catalytic subunits. J Biol Chem 267:687-690.

Arshavsky VY (2002) Rhodopsin phosphorylation: from terminating single photon responses to photoreceptor dark adaptation. Trends Neurosci 25:124-126.

Arshavsky VY, Lamb TD, Pugh EN Jr (2002) G proteins and phototransduction. Annu Rev Physiol 64:153-187.

Artemyev NO (2008) Light-dependent compartmentalization of transducin in rod photoreceptors. Mol Neurobiol 37:44-51.

Binder BM, Biernbaum MS, Bownds MD (1990) Light activation of one rhodopsin molecule causes the phosphorylation of hundreds of others. A reaction observed in electropermeabilized frog rod outer segments exposed to dim illumination. J Biol Chem 265:15333-15340.

Brann MR, Cohen LV (1987) Diurnal expression of transducin mRNA and translocation of transducin in rods of rat retina. Science 235:585-587.

Burns ME, Arshavsky VY (2005) Beyond counting photons: trials and trends in vertebrate visual transduction. Neuron 48:387-401.

Calvert PD, Strissel KJ, Schiesser WE, Pugh EN Jr, Arshavsky VY (2006) Light-driven translocation of signaling proteins in vertebrate photoreceptors. Trends Cell Biol 16:560-568.

Chen CK, Burns ME, Spencer M, Niemi GA, Chen J, Hurley JB, Baylor DA, Simon MI (1999) Abnormal photoresponses and light-induced apoptosis in rods lacking rhodopsin kinase. Proc Natl Acad Sci U S A 96:3718-3722.

Chen CK, Burns ME, He W, Wensel TG, Baylor DA, Simon MI (2000) Slowed recovery of rod photoresponse in mice lacking the GTPase accelerating protein RGS9-1. Nature 403:557-560.

Chen J, Wu M, Sezate SA, McGinnis JF (2007) Light threshold-controlled cone alpha-transducin translocation. Invest Ophthalmol Vis Sci 48:3350-3355.

Coleman JE, Semple-Rowland SL (2005) GC1 deletion prevents lightdependent arrestin translocation in mouse cone photoreceptor cells. Invest Ophthalmol Vis Sci 46:12-16.

Cowan CW, Fariss RN, Sokal I, Palczewski K, Wensel TG (1998) High expression levels in cones of RGS9, the predominant GTPase accelerating protein of rods. Proc Natl Acad Sci U S A 95:5351-5356.

Daniele LL, Lillo C, Lyubarsky AL, Nikonov SS, Philp N, Mears AJ, Swaroop A, Williams DS, Pugh EN Jr (2005) Cone-like morphological, molecular, and electrophysiological features of the photoreceptors of the $\mathrm{Nrl}$ knockout mouse. Invest Ophthalmol Vis Sci 46:2156-2167.

Deng WT, Sakurai K, Liu J, Dinculescu A, Li J, Pang J, Min SH, Chiodo VA, Boye SL, Chang B, Kefalov VJ, Hauswirth WW (2009) Functional inter- changeability of rod and cone transducin alpha-subunits. Proc Natl Acad Sci U S A 106:17681-17686.

Elias RV, Sezate SS, Cao W, McGinnis JF (2004) Temporal kinetics of the light/dark translocation and compartmentation of arrestin and alphatransducin in mouse photoreceptor cells. Mol Vis 10:672-681.

Fain GL (2006) Why photoreceptors die (and why they don't). BioEssays 28:344-354.

Fu Y, Yau KW (2007) Phototransduction in mouse rods and cones. Pflugers Arch 454:805-819.

Gurevich VV, Gurevich EV (2003) The new face of active receptor bound arrestin attracts new partners. Structure 11:1037-1042.

Imai H, Kojima D, Oura T, Tachibanaki S, Terakita A, Shichida Y (1997) Single amino acid residue as a functional determinant of rod and cone visual pigments. Proc Natl Acad Sci U S A 94:2322-2326.

Kawamura S, Tachibanaki S (2008) Rod and cone photoreceptors: molecular basis of the difference in their physiology. Comp Biochem Physiol A Mol Integr Physiol 150:369-377.

Kefalov V, Fu Y, Marsh-Armstrong N, Yau KW (2003) Role of visual pigment properties in rod and cone phototransduction. Nature 425:526531.

Kennedy MJ, Lee KA, Niemi GA, Craven KB, Garwin GG, Saari JC, Hurley JB (2001) Multiple phosphorylation of rhodopsin and the in vivo chemistry underlying rod photoreceptor dark adaptation. Neuron 31:87-101.

Kennedy MJ, Dunn FA, Hurley JB (2004) Visual pigment phosphorylation but not transducin translocation can contribute to light adaptation in zebrafish cones. Neuron 41:915-928.

Keresztes G, Martemyanov KA, Krispel CM, Mutai H, Yoo PJ, Maison SF, Burns ME, Arshavsky VY, Heller S (2004) Absence of the RGS9.Gbeta5 GTPase-activating complex in photoreceptors of the R9AP knockout mouse. J Biol Chem 279:1581-1584.

Kerov V, Chen D, Moussaif M, Chen YJ, Chen CK, Artemyev NO (2005) Transducin activation state controls its light-dependent translocation in rod photoreceptors. J Biol Chem 280:41069-41076.

Kosloff M, Alexov E, Arshavsky VY, Honig B (2008) Electrostatic and lipid anchor contributions to the interaction of transducin with membranes: mechanistic implications for activation and translocation. J Biol Chem 283:31197-31207.

Krispel CM, Sokolov M, Chen YM, Song H, Herrmann R, Arshavsky VY, Burns ME (2007) Phosducin regulates the expression of transducin $\beta \gamma$ subunits in rod photoreceptors and does not contribute to phototransduction adaptation. J Gen Physiol 130:303-312.

Li Q, Timmers AM, Guy J, Pang J, Hauswirth WW (2008) Cone-specific expression using a human red opsin promoter in recombinant AAV. Vision Res 48:332-338.

Lobanova ES, Finkelstein S, Song H, Tsang SH, Chen CK, Sokolov M, Skiba NP, Arshavsky VY (2007) Transducin translocation in rods is triggered by saturation of the GTPase-activating complex. J Neurosci 27:1151-1160.

Lobanova ES, Finkelstein S, Herrmann R, Chen YM, Kessler C, Michaud NA, Trieu LH, Strissel KJ, Burns ME, Arshavsky VY (2008) Transducin $\gamma$-subunit sets expression levels of $\alpha$ - and $\beta$-subunits and is crucial for rod viability. J Neurosci 28:3510-3520.

Lyubarsky AL, Falsini B, Pennesi ME, Valentini P, Pugh EN Jr (1999) UVand midwave-sensitive cone-driven retinal responses of the mouse: a possible phenotype for coexpression of cone photopigments. J Neurosci 19:442-455.

Lyubarsky AL, Chen C, Simon MI, Pugh EN Jr (2000) Mice lacking G-protein receptor kinase 1 have profoundly slowed recovery of conedriven retinal responses. J Neurosci 20:2209-2217.

Lyubarsky AL, Naarendorp F, Zhang X, Wensel T, Simon MI, Pugh EN Jr (2001) RGS9-1 is required for normal inactivation of mouse cone phototransduction. Mol Vis 7:71-78.

Maeda T, Imanishi Y, Palczewski K (2003) Rhodopsin phosphorylation: 30 years later. Prog Retin Eye Res 22:417-434.

Martemyanov KA, Krispel CM, Lishko PV, Burns ME, Arshavsky VY (2008) Functional comparison of RGS9 splice isoforms in a living cell. Proc Natl Acad Sci U S A 105:20988-20993.

Mears AJ, Kondo M, Swain PK, Takada Y, Bush RA, Saunders TL, Sieving PA, Swaroop A (2001) Nrl is required for rod photoreceptor development. Nat Genet 29:447-452.

Mustafi D, Engel AH, Palczewski K (2009) Structure of cone photoreceptors. Prog Retin Eye Res 28:289-302. 
Neubert TA, Johnson RS, Hurley JB, Walsh KA (1992) The rod transducin alpha subunit amino terminus is heterogeneously fatty acylated. J Biol Chem 267:18274-18277.

Nikonov SS, Daniele LL, Zhu X, Craft CM, Swaroop A, Pugh EN Jr (2005) Photoreceptors of Nrl-/- mice coexpress functional S- and M-cone opsins having distinct inactivation mechanisms. J Gen Physiol 125:287-304.

Nikonov SS, Kholodenko R, Lem J, Pugh EN Jr (2006) Physiological features of the S- and M-cone photoreceptors of wild-type mice from singlecell recordings. J Gen Physiol 127:359-374.

Nikonov SS, Brown BM, Davis JA, Zuniga FI, Bragin A, Pugh EN Jr, Craft CM (2008) Mouse cones require an arrestin for normal inactivation of phototransduction. Neuron 59:462-474.

Philp NJ, Chang W, Long K (1987) Light-stimulated protein movement in rod photoreceptor cells of the rat retina. FEBS Lett 225:127-132.

Rodieck RW (1998) The first steps in seeing. Sunderland, MA: Sinauer Associates.

Rosenzweig DH, Nair KS, Wei J, Wang Q, Garwin G, Saari JC, Chen CK, Smrcka AV, Swaroop A, Lem J, Hurley JB, Slepak VZ (2007) Subunit dissociation and diffusion determine the subcellular localization of rod and cone transducins. J Neurosci 27:5484-5494.

Sakurai K, Onishi A, Imai H, Chisaka O, Ueda Y, Usukura J, Nakatani K, Shichida Y (2007) Physiological properties of rod photoreceptor cells in green-sensitive cone pigment knock-in mice. J Gen Physiol 130:21-40.

Saszik SM, Robson JG, Frishman LJ (2002) The scotopic threshold response of the dark-adapted electroretinogram of the mouse. J Physiol 543:899916.

Sharma S, Ball SL, Peachey NS (2005) Pharmacological studies of the mouse cone electroretinogram. Vis Neurosci 22:631-636.

Shi G, Yau KW, Chen J, Kefalov VJ (2007) Signaling properties of a shortwave cone visual pigment and its role in phototransduction. J Neurosci 27:10084-10093

Shichida Y, Imai H, Imamoto Y, Fukada Y, Yoshizawa T (1994) Is chicken green-sensitive cone visual pigment a rhodopsin-like pigment? A comparative study of the molecular properties between chicken green and rhodopsin. Biochemistry 33:9040-9044.

Sitaramayya A, Liebman PA (1983) Phosphorylation of rhodopsin and quenching of cyclic GMP phosphodiesterase activation by ATP at weak bleaches. J Biol Chem 258:12106-12109.

Skiba NP, Bae H, Hamm HE (1996) Mapping of effector binding sites of transducin alpha-subunit using G $\alpha$ t/G $\alpha$ il chimeras. J Biol Chem 271:413-424.

Skiba NP, Hopp JA, Arshavsky VY (2000) The effector enzyme regulates the duration of $G$ protein signaling in vertebrate photoreceptors by increasing the affinity between transducin and RGS protein. J Biol Chem 275:32716-32720

Slepak VZ, Hurley JB (2008) Mechanism of light-induced translocation of arrestin and transducin in photoreceptors: interaction-restricted diffusion. IUBMB Life 60:2-9.

Sokolov M, Lyubarsky AL, Strissel KJ, Savchenko AB, Govardovskii VI, Pugh EN Jr, Arshavsky VY (2002) Massive light-driven translocation of transducin between the two major compartments of rod cells: a novel mechanism of light adaptation. Neuron 34:95-106.

Sokolov M, Strissel KJ, Leskov IB, Michaud NA, Govardovskii VI, Arshavsky VY (2004) Phosducin facilitates light-driven transducin translocation in rod photoreceptors. Evidence from the phosducin knockout mouse. J Biol Chem 279:19149-19156.

Tachibanaki S, Tsushima S, Kawamura S (2001) Low amplification and fast visual pigment phosphorylation as mechanisms characterizing cone photoresponses. Proc Natl Acad Sci U S A 98:14044-14049.

Tachibanaki S, Arinobu D, Shimauchi-Matsukawa Y, Tsushima S, Kawamura S (2005) Highly effective phosphorylation by G protein-coupled receptor kinase 7 of light-activated visual pigment in cones. Proc Natl Acad Sci U S A 102:9329-9334.

Timmers AM, Zhang H, Squitieri A, Gonzalez-Pola C (2001) Subretinal injections in rodent eyes: effects on electrophysiology and histology of rat retina. Mol Vis 7:131-137.

Weiss ER, Ducceschi MH, Horner TJ, Li A, Craft CM, Osawa S (2001) Species-specific differences in expression of G-protein-coupled receptor kinase (GRK) 7 and GRK1 in mammalian cone photoreceptor cells: implications for cone cell phototransduction. J Neurosci 21:9175-9184.

Whelan JP, McGinnis JF (1988) Light-dependent subcellular movement of photoreceptor proteins. J Neurosci Res 20:263-270.

Xu J, Dodd RL, Makino CL, Simon MI, Baylor DA, Chen J (1997) Prolonged photoresponses in transgenic mouse rods lacking arrestin. Nature 389:505-509.

Yokoyama R, Yokoyama S (2000) Comparative molecular biology of visual pigments. In: Molecular mechanisms in visual transduction (Stavenga DG, De Grip WJ, Pugh EN, eds), pp 257-296. New York: Elsevier.

Zhang X, Wensel TG, Kraft TW (2003) GTPase regulators and photoresponses in cones of the eastern chipmunk. J Neurosci 23:1287-1297.

Zolotukhin S, Potter M, Zolotukhin I, Sakai Y, Loiler S, Fraites TJ Jr, Chiodo VA, Phillipsberg T, Muzyczka N, Hauswirth WW, Flotte TR, Byrne BJ Snyder RO (2002) Production and purification of serotype 1, 2, and 5 recombinant adeno-associated viral vectors. Methods 28:158-167. 